\title{
Are Visual Texture-selective Areas Recruited During Haptic Texture Discrimination?
}

Samantha K. Podrebarac, The University of Western Ontario

Supervisor: Melvyn Goodale, The University of Western Ontario

A thesis submitted in partial fulfillment of the requirements for the Master of Science degree in Neuroscience

(C) Samantha K. Podrebarac 2013

Follow this and additional works at: https://ir.lib.uwo.ca/etd

Part of the Cognition and Perception Commons, and the Cognitive Psychology Commons

\section{Recommended Citation}

Podrebarac, Samantha K., "Are Visual Texture-selective Areas Recruited During Haptic Texture Discrimination?" (2013). Electronic Thesis and Dissertation Repository. 1437.

https://ir.lib.uwo.ca/etd/1437

This Dissertation/Thesis is brought to you for free and open access by Scholarship@Western. It has been accepted for inclusion in Electronic Thesis and Dissertation Repository by an authorized administrator of Scholarship@Western. For more information, please contact wlswadmin@uwo.ca. 


\section{ARE VISUAL TEXTURE-SELECTIVE AREAS RECRUITED DURING HAPTIC TEXTURE DISCRIMINATION?}

(Thesis Format: Integrated Article)

by

Samantha K. Podrebarac

Graduate Program in Neuroscience

A thesis submitted in partial fulfillment

of the requirements for the degree of Master of Science

The School of Graduate and Postdoctoral Studies

The University of Western Ontario

London, Ontario, Canada

(c) Samantha K. Podrebarac 2013 


\begin{abstract}
Shape and texture provide cues to object identity, both when objects are explored using vision and via touch (haptics). Visual shape information is processed within the lateral occipital complex (LOC), while texture is processed in medial regions of the collateral sulcus $(\mathrm{CoS})$. Evidence indicates that the LOC is consistently recruited during both visual and haptic shape processing. We used functional magnetic resonance imaging (fMRI) to examine whether 'visual' texture-selective areas are similarly recruited when observers discriminate texture via touch. We used a blocked design in which participants attended to either the texture or shape of a number of 3-dimensional (3D) objects, via vision or touch. We observed significant haptic texture-selective fMRI responses in medial occipitotemporal cortex within areas adjacent to, but not overlapping, those recruited during visual texture discrimination. Our data demonstrate that occipitotemporal areas typically associated with visual processing are recruited during the perception of surface texture via touch.
\end{abstract}

Keywords: Haptics, vision, texture, shape, collateral sulcus, lateral occipital complex. 


\section{Acknowledgements}

I would like to thank my supervisors, Mel Goodale and Jacquie Snow. The two of you make an exceptional teaching team and your guidance is the sole reason that I was able to learn so much over the past two years. I am incredibly lucky to have had the privilege to work with both of you and I want to thank you for providing me with support while at the same time giving me the freedom to take the lead on this project. This opportunity has been influential to my growth as a young researcher.

I would like to acknowledge the team that helped with my project as this would not have been a success without their assistance. Thank you to Adam McLean, Haitao Yang, Derek Quinlan, Portia Tsoi, Jim Ladich, and Oksana Opalevych.

Special thank you to my friends and family for supporting and encouraging me in everything that I do - my success would not be possible without them. 


\section{TABLE OF CONTENTS}

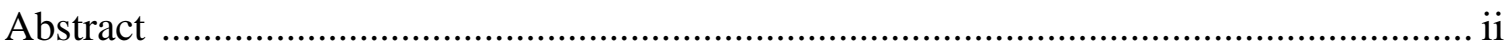

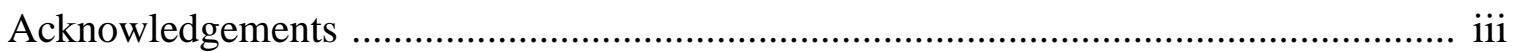

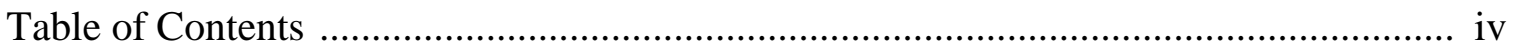

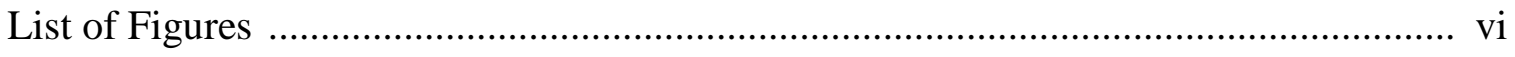

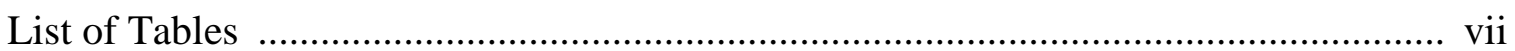

\section{Chapter 1}

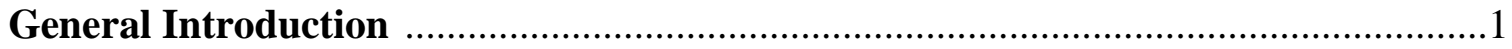

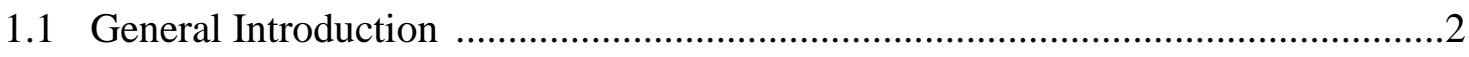

1.1.1 Visual Object Perception .................................................................2

1.1.2 Relationship Between Vision and Haptics ...............................................4

1.1.3 Haptic object perception ..................................................................

1.1.4 Haptic texture perception ...........................................................6

1.1.5 Motivation for the Current Experiment ……….......................................

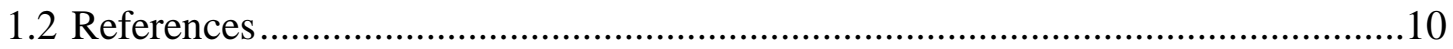

\section{Chapter 2}

\section{Are Visual Texture-selective Areas Recruited During Haptic Texture}

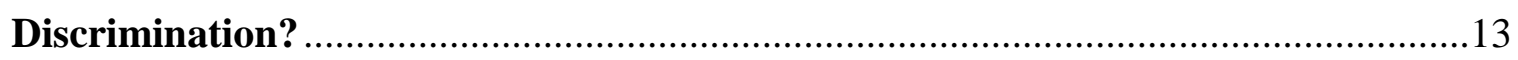

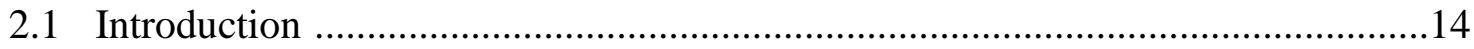

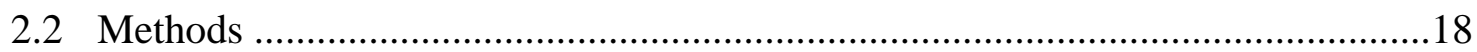

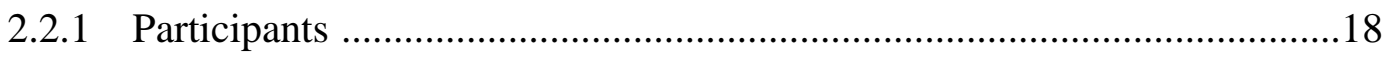




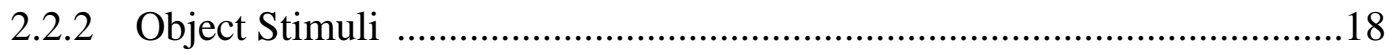

2.2.3 Experimental Procedure and Design .......................................................20

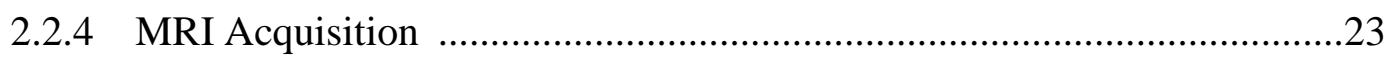

2.2.5 fMRI Data Pre-processing and Analysis ................................................23

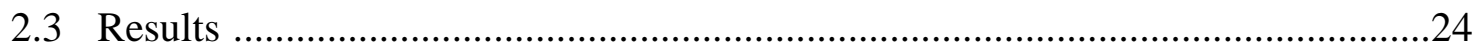

2.3.1 Whole Brain Random Effects Analysis ………………………..............24

2.3.2 Group-level ROI analyses .................................................................

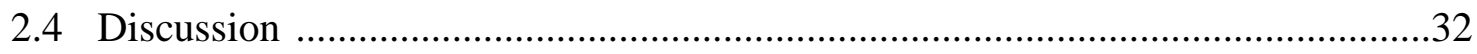

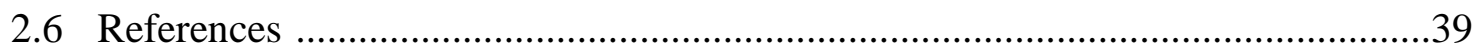

\section{Chapter 3}

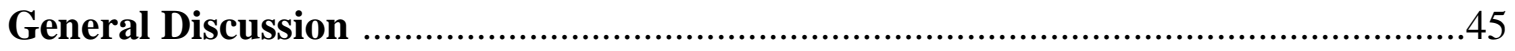

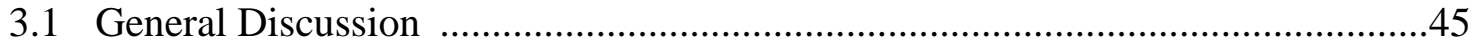

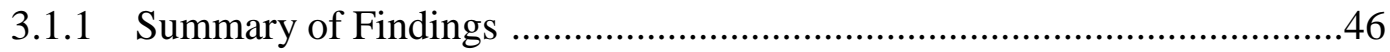

3.1.2 Haptic and visual processing of object texture …....................................46

3.1.3 Evidence for separate pathways for haptic surface texture vs shape

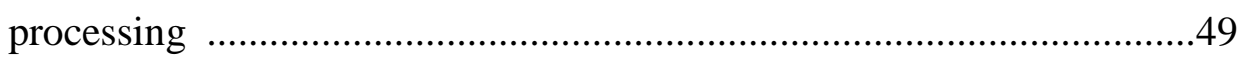

3.1.4 Relation of the findings to previous research on visual form and texture

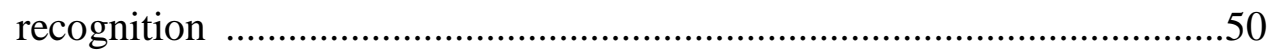

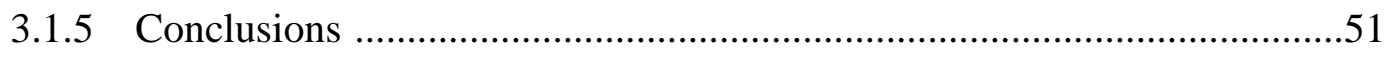

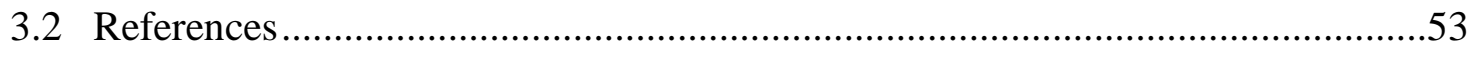

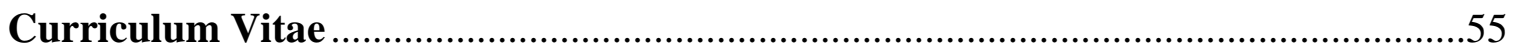




\section{List of Figures}

Figure 1 Experimental set up and stimuli exemplars ..................................... 19

Figure 2 Experimental paradigm …........................................................ 21

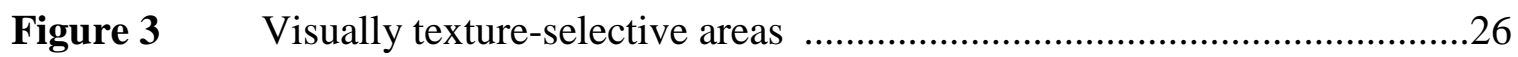

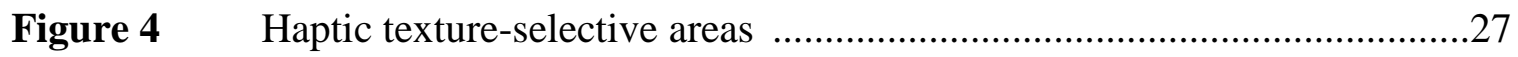

Figure 5 Haptic and visual texture activation at a threshold of $\mathrm{p}<0.01 \quad \ldots \ldots \ldots \ldots . . .27$

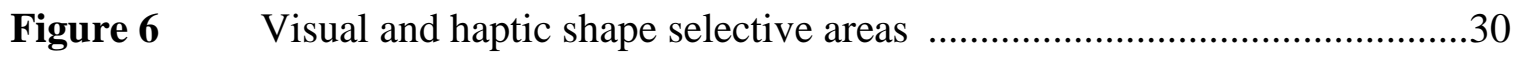




\section{List of Tables}

Table 1 Coordinates of the regions that activated for visual-texture and

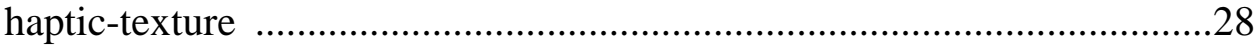

Table 2 Coordinates of the regions that activated for visual-shape and

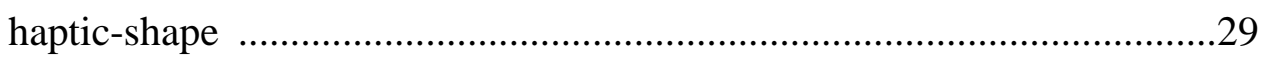


Chapter 1

\section{General Introduction}




\subsection{GENERAL INTRODUCTION}

The ability to perceive and recognize objects is essential to human survival, and most often, we perceive objects through vision before any other sense. In fact, vision is the dominant modality for object perception. Typically, we reach out to feel an object's weight or texture only after we have seen it. Nevertheless, the relationship between vision and touch (haptics) is unique in that these two senses allow us to most easily access three-dimensional (3D) object information (James et al., 2006).

\subsubsection{Visual object perception}

Humans are able to distinguish objects based on the perception of their features, including size, color, shape, and texture. Recent work has demonstrated that visual regions of the brain have a unique cortical organization whereby different object properties including texture, form, color, and facial cues are encoded in specific regions of the ventral-stream (Peuskens et al., 2004; Cant and Goodale, 2007; Cant et al., 2009; Cavina-Pratesi et al., 2010; Kourtzi and Kanwisher, 2001; Allison et al., 1994; Kanwisher et al., 1997).

Cant and Goodale (2007; Cant et al., 2009) found that within the visual domain, shape is processed in the lateral occipital cortex (LOC) (Kourtzi and Kanwisher, 2001), while texture is processed along the collateral sulcus (CoS) (Puce et al., 1996; Peuskens et al., 2004). In their studies Cant et al. presented images of textured 3D shapes to 
observers within an fMRI scanner. Participants were instructed to make a same-different judgment with regard to either the form, texture, or color of each object image. Analysis of within-subjects effects revealed that form perception activated more lateral regions (LOC) and texture perception activated more medial regions (including CoS). Color perception did not activate any ventral stream regions, but rather, was found in a more posterior region of V1 and in the cuneus. Cant et al.'s work indicates that visual perceptions of form engages the lateral regions of occipitotemporal cortex, while texture perception engages comparatively more medial regions of occipitotemporal cortex (Cant and Goodale, 2007; Cant et al., 2009).

Cant et al.'s $(2007,2009)$ results are supported by research in neuropsychological patient populations. For example, Cavina-Pratesi et al. (2010) studied two patients who have a complementary lesion pattern. Patient D.F. has an intact $\operatorname{CoS}$ but lesions to her LOC and is therefore unable to perceive the shape of objects. Conversely, patient M.S. has an intact LOC, but lesioned $\mathrm{CoS}$ - and although he remains able to perceive object shape, he cannot perceive surface texture visually. Cavina-Pratesi et al., (2010) conducted an fMRI study whereby patient D.F, M.S., and a control group were presented with 3dimensional (3D) shaded greyscale object images. In one condition, participants were asked to indicate which one of three different shapes was different from the other two. In another condition, participants were presented with three identical shapes (spheres) that each differed in their surface texture. In this condition, observers were required to pick the 'odd-man out' in terms of surface texture. Analysis of the imaging data revealed that D.F. showed significant activation in the CoS regions during the texture-variant trials, 
however she failed to show any activation in the vicinity of form-selective LOC when attempting to perceive the shape-variant trials. Conversely, M.S. showed an inverse activation pattern - activity in LOC during shape-variant trials, but no activation in CoS during texture-variant trials. The behavioral data also confirmed the imaging results: D.F. performed well on texture-discrimination trials and at chance on the shape-discrimination task, while M.S. showed the reverse pattern.

Taken together, these data suggest a broad organizational pattern in which the visual system is divided into two streams for visual object processing whereby posteriorlateral regions of occipitotemporal cortex (LOC) are involved in the perception of object shape, whereas more medial regions, such as the parahippocampal gyrus (also referred to as the collateral sulcus $(\mathrm{CoS}))$, the inferior occipital gyrus (IOG), and the lingual gyrus (LG) (Peuskins et al., 2004; Cant and Goodale, 2007; Cavina-Pratesi et al., 2010) are involved in the visual perception of texture.

\subsubsection{Relationship between vision and haptics}

The vast majority of imaging research surrounding object perception has involved the visual perception of two-dimensional (2D) images of objects. Though we may perceive objects most often through vision alone, we are also able to perceive objects through other sensory domains including touch and audition. There is an undoubtedly a strong link between the way that we perceive objects through vision and through touch (haptics). The hierarchical organization of these two senses is also comparable in that 
early perception starts at highly specific sites with small receptive fields (i.e., the fingertips or the fovea) and progresses to higher-level cortical areas with larger receptive fields (ie. somatosensory cortex or occipital regions) (Farah, 2000; Goldstein, 2001). Moreover, vision and haptics often occur together, and perception in one modality has a tendency to motivate perception in the other (James et al., 2006). For instance, when we see a textured object, we are likely to reach out and palpate it. The link between haptics and vision is important in understanding the differences between unimodal and multimodal object representations, and how these representations are stored in the brain.

\subsubsection{Haptic object perception}

The extraction of shape information through haptic perception is an important component of object recognition. Examinations of haptic perception have found that visually shape-selective regions of the ventral stream (LOC) are also activated when participants investigate object shape via touch (Amedi et al., 2001; Allen and Humphreys, 2009; Amedi et al., 2002; Grefkes et al., 2002; James et al., 2002; Naumer et al., 2010; Pietrini et al., 2004; Stilla and Sathian, 2008; Tal and Amedi, 2009; Zhang et al., 2004). Importantly, Amedi et al. (2001) asked participants both to palpate a number of objects and view images of the same objects while inside the fMRI scanner. They found that a sub-region of the LOC, (which they later termed the lateral occipital tactile visual area (LOtv) (Amedi et al., 2002), activated during both visual and tactile object perception. They concluded that the LOC was a critical bimodal region responsible for both haptic and visual shape representation (James et al., 2002). 
Tal and Amedi (2009) examined whether the visuo-haptic fMRI responses observed within the LOtv reflected a multisensory representation (i.e., from multisensory neurons), or a population of inter-digitated, but functionally independent haptic and visual neurons that encoded unimodal representations. They examined this question using fMR-adaptation (fMR-A), and found adaptation from visual object perception to tactile object perception in LOtv - a result that they argued was suggestive of a shared underlying neural basis for visual and haptic perception (Tal and Amedi, 2009).

James et al. (2007) have also argued persuasively that the behavioral effects of patient D.F.'s lesion in LOC are supported by the bimodal visual and haptic activity in LOtv found by Amedi and colleagues (2001; 2002; 2009). D.F. is unable to perceive object shape through haptics or via vision. The haptic impairment caused by her LOC lesion is suggestive of the fact that the LOC is not only necessary for perception of object shape via vision, but also via touch.

Given that texture is an object property, similar to shape, that is salient to both the visual and haptic domains, only recently, have groups started to examine the neural basis of haptic texture perception and its relation to vision.

\subsubsection{Haptic texture perception}

Texture perception via touch is an important adjunct to object perception and recognition. The texture of an object can provide crucial information about its identity; 
for instance, though the shape of a golf ball and a ping-pong ball are identical, they can be distinguished by their textural differences. Haptic perception of texture is integral to object recognition because texture is a property that tends to be most salient to touch, above all other senses (Klatzky et al., 1987).

The cortical basis of texture perception is considerably different between vision and touch. As outlined earlier, the visual perception of texture involves early visual areas of the brain, regions of the CoS, the IOG, and the LG (Peuskens et al., 2004; Cant and Goodale, 2007; Cant et al., 2009). The haptic perception of texture, on the other hand, has been consistently found to involve regions of somatosensory cortex (I and II) and insular regions (for review see James et al., 2007). The vast majority of these studies, however, have focused on mapping the neural correlates of shape and texture perception unimodally.

A study by Stilla and Sathian (2008) examined the perception of both haptic and visual texture using fMRI. Participants were instructed to either view or palpate the texture or shape of a number of stimuli. Importantly, they used two different sets of stimuli for haptic and visual perception. During texture perception, they reported overlap in the medial occipital cortex (MOC) when participants were both palpating for object texture and when they were viewing object texture. Upon further examination of the activity, this group failed to report correlated activity between vision and touch in the MOC, thereby prompting the authors to postulate that the MOC contains subpopulations of visual neurons and haptic neurons. This suggests that the representation of texture 
derived from haptics and from vision may be distinct, though due to the fact that different stimuli were used in both conditions, further investigation is required.

Sathian and colleagues (2011) also conducted an imaging study to examine the haptic and visual perception of location and textural information. This group found haptic-texture selectivity again in the parietal operculum (supporting their 2008 study), within the posterior visual cortex, and in the left inferior frontal cortex. They also found bimodal texture-related activity in regions of the posterior visual, left inferior frontal cortex, and middle occipital gyrus (supporting their 2008 study). Again, this group used a separate set of stimuli in their visual-texture condition as compared to their haptic-texture condition. The haptic stimuli consisted of fabric or upholstery glued to pieces of cardboard, while the visual stimuli were photographs of the same fabrics from the haptic condition. Importantly, the objects were placed in the participants' right hand during trials, which could potentially incorporate involuntary processing of other material properties such as weight or even introduce involuntary processing of shape properties since the textures were glued to 3D cardboard cutouts.

The visuo-haptic MOG overlap is interesting in light of results reported by James et al. (2002), who found evidence for visuo-haptic cross modal priming in this same region during a task that involved variation in object shape but not texture. It is therefore possible that the bimodal MOG reported by Stilla and Sathian $(2008 ; 2011)$ reflects a shared processing of object shape rather than texture. 


\subsubsection{Motivation for the Current Experiment}

The goal of my thesis was to determine whether regions of the collateral sulcus that have been repeatedly implicated in the processing of visual representations of texture, are also active when participants perceive the same textures via touch. To answer this question, I conducted an fMRI study whereby participants were asked to attend to the "texture" or "shape" of a number of objects either via vision or via touch. I first contrasted activation when participants were attending to the texture of objects with the activation while they attended to the shape of objects, in order to isolate regions that showed more activation to texture perception than to shape perception (texture > shape). I ran the latter contrast in each modality (vision and touch) separately, then compared the locations of texture-selective activation. The reverse contrast was also run (shape > texture) in each modality separately to isolate shape-selective regions. Importantly, I opted to use identical 3D stimuli for both haptic and visual perception runs, in order to equate the two conditions so that I could fairly compare activation between the two sensory modalities. 


\subsection{REFERENCES}

Allen, H.A. and Humphreys, G.W. (2009). Direct tactile stimulation of dorsal occipitotemporal cortex in a visual agnosic. Current Biology, 19(12) 1044 - 1049.

Allison, T., McCarthy, G., Nobre, A., Puce, A., Belger, A. (1994). Human extrastriate visual cortex and the perception of faces, words, numbers, and colors. Cerebral Cortex, 4:544-554.

Amedi, A., Malach, R., Hendle, T., Peled, S., and Zohary, E. (2001). Visuo-haptic objectrelated activation in the ventral visual pathway. Nature Neuroscience. 4:324-330.

Amedi, A., Jacobson, G., Hendler, T., Malach, R., and Zohary, E. (2002). Convergence of visual and tactile shape processing in the human lateral occipital complex. Cereb Cortex, 12:1202-1212.

Cant JS, Goodale MA. Attention to form or surface properties modulates different regions of human occipitotemporal cortex. Cereb. Cortex. 2007;17:713-731

Cant, J.S., Arnott, S.R., and Goodale, M.A. (2009). fMR-adaptation reveals separate processing regions for the perception of form and texture in the human ventral stream. Exp. Brain Res., 192:391-405.

Cavina-Pratesi, C., Kentridge, R.W., Heywood, C.A., and Milner, A.D. (2010). Separate channels for processing form, texture, and color: Evidence from fMRI adaptation and visual object agnosia (2010). Cerebral Cortex, 20 (10), 2319-2332.

Farah, M.J. (2000). The cognitive neuroscience of vision. Fundamentals of cognitive neuroscience. Malden: Blackwell Publishing.

Goldstein, E. B. (2001). Sensation and perception, $6^{\text {th }}$ edition. Belmont, CA: Wadsworth 
Publishing Company.

Grefkes, C., Weiss, P.H., Zilles, K., Fink, G.R., 2002. Crossmodal processing of object features in human anterior intraparietal cortex, an fMRI study implies equivalencies between humans and monkeys. Neuron 35, 173-184.

James, T.W., Humphrey, G.K., Gati, J.S., Servos, P., Menon, R.S., and Goodale, M.A. (2002). Haptic study of three-dimensional objects activates extrastriate visual areas. Neuropsychologia, 40, 1706-1714.

James, T.W., James, K.H., Humphrey, G.K., \& Goodale, M.A. (2006). Do visual and tactile object representations share the same neural substrate? In M.A. Heller and S. Ballesteros (Eds.), Touch and blindness: psychology and neuroscience. Mahwah, NJ: Lawrence Erlbaum.

James, T.W., Kim, S., and Fisher, J.S. (2007). The neural basis of haptic object processing. Can Journ of Exp Psych. 61 (3), 219-229.

Kanwisher, N. G., McDermott, J., \& Chun, M. M. (1997). The fusiform face area: a module in human extrastriate cortex specialized for face perception. Journal of Neuroscience, 17, 4302-4311.

Klatzky, R.L., Lederman, S.J., and Reed, C.L. (1987). Theres more to touch than meets the eye: the salience of object attributes for haptics with and without vision. $J$. Exp. Psychol. Gen. 116, 356-369.

Kourtzi, Z. and Kanwisher, N. (2001). Representation of perceived object shape by the human lateral occipital complex. Science. 293, 1506-1509. 
Naumer, M.J., Ratz, L., Yalachkov, Y., Polony, A., Doehrmann, O., van de Ven, V., Muller, N.G., Kaiser, J., and Hein, G. (2010). Visuohaptic convergence in a corticocerebellar network. European Journal of Neuroscience, 1-7.

Peuskens, H., Claeys, K.G., Todd, J.T., Norman, J.F., Van Hecke, P., and Orban, G.A. (2004). Attention to 3-D shape, 3-D motion, and texture in 3-D structure from motion displays. J. Cognit. Neurosci. 16:665-682.

Pietrini, P., Furey, M.L., Ricciardi, E. et al (2004). Beyond sensory images: object-based representation in the human ventral pathway. Proc Natl Acad Sci USA 101:56585663.

Puce, A., Allison, T., Asgari, M., Gore, J.C., and McCarthy, G. (1996). Differential sensitivity of human visual cortex to faces, letterstrings and textures: a functional magnetic resonance imaging study. J. Neurosci. 16:5005-5215.

Sathian, K., Lacey, S., Stilla, R., Gibson, G.O., Deshpanae, G., Hu, X., LaConte, S., and Glielmi, C. (2011). Dual pathways for haptic and visual perception of spatial and texture information. Neuroimage, 57, 462-475.

Stilla R. and Sathian K. (2008). Selective visuo-haptic processing of shape and texture Hum. Brain Mapp. 2008;29:1123-1138.

Tal, N. and Amedi, A. (2009). Multisensory visual-tactile object related network in humans: insights gained using a novel crossmodal adaptation approach. Exp Brain Res., 198 (2-3): 165-182.

Zhang, M., Weisser, V.D., Stilla, R., Prather, S.C., and Sathian, K. (2004) Multisensory cortical processing of object shape and its relation to mental imagery. Cogn Affect Behav Neurosci, 4:251-259. 


\section{Chapter 2}

\section{Are Visual Texture-selective Areas Recruited \\ During Haptic Texture Discrimination?}




\subsection{INTRODUCTION}

The perception of shape is an important factor in the identification of objects. At the same time, surface properties, such as colour and texture, are also critical. Moreover, we can access information about shape and surface properties such as texture through both vision and touch.

The vast majority of imaging research studying object shape has focused on localizing regions of the brain that are involved in its visual perception. One of the areas that has consistently been implicated is the lateral occipital cortex (LOC; for review see Grill-Spector and Malach, 2004). Although there have also been many imaging studies of colour perception (for review, see Tanaka et al., 2001), the visual perception of surface texture has received far less attention. Recently, however, Cant and Goodale (2007) have shown that medial, rather than lateral, regions of the ventral visual stream are involved in the visual perception of surface texture. This finding converges with earlier observations by Peuskens and colleagues (2004). Specifically, Cant demonstrated that areas within the collateral sulcus $(\mathrm{CoS})$ and other more medial areas of the ventral stream are involved in the visual perception of texture, while more lateral regions, including LOC, are involved in the visual perception of shape (Cant and Goodale 2007; Cant et al., 2009).

Behavioral and imaging studies with patient populations also provide support for the involvement of medial regions of occipitotemporal cortex in visual texture perception and more lateral brain regions in visual shape perception. Cavina-Pratesi and colleagues 
(2010) examined the perception of texture and shape in two patients; D.F., who has a bilateral lesion in the lateral occipital cortex of her brain but no damage to medial occipitotemporal regions, and patient M.S. who shows the inverse lesion pattern to D.F. intact LOC, but bilateral lesions in the medial occipital areas. When attending to visual texture, D.F. showed fMRI activation in medial occipital areas, such as the $\mathrm{CoS}-$ the same region where M.S.'s lesion was located. Conversely, while attending to shape, M.S. showed activation in LOC, where D.F.'s lesion is located. Behavioral results converged nicely with the activation pattern: D.F. performed well on texture discrimination and at chance on the shape task, whereas M.S. showed the reverse pattern.

Interestingly, there is accumulating evidence from fMRI that laterally-located 'visual' shape-selective areas are also invoked during haptic shape processing (Allen and Humphreys, 2009; Amedi et al., 2002; Grefkes et al., 2002; James et al., 2002; Naumer et al., 2010; Pietrini et al., 2004; Stilla and Sathian, 2008; Tal and Amedi, 2009; Zhang et al., 2004). Most notably, Amedi and colleagues (Amedi et al., 2001; Amedi et al., 2002) demonstrated that a sub-region of the LOC, which they termed the lateral occipital tactile-visual area (LOtv), was activated both when participants palpated objects with different shapes and when they viewed the different objects. These findings have prompted the dominant view that the lateral occipital area constitutes a region of multisensory cortex, facilitating visuo-haptic shape representation (Amedi et al., 2002; Amedi et al., 2005; Sathian et al., 2011; Lacey et al., 2009; Lucan et al., 2010). Here, we asked an analogous question, of whether medially-located areas implicated in the visual recognition of surface texture are also recruited during haptic exploration of the surface 
of objects. Although there have been a number of imaging studies that have examined haptic texture identification, these studies have focused largely on tactile routines (Simoes-Franklin et al., 2011) and somatosensory neural correlates of unimodal tactile perception (O'Sullivan et al., 1994; Servos et al., 2001; Nelson et al., 2004; for review see Kaas \& Collins, 2003).

A study by Stilla and Sathian (2008) found bilateral haptic-only texture-selectivity in the parietal operculum and posterior insula, in addition to overlapping haptic and visual texture-activation in the right medial occipital cortex (MOC). This group argued that an absence of correlation between fMRI responses during visual vs. haptic texture perception in the MOC reflected the existence of unimodal neurons, rather than multisensory cortex. In a later examination of visual and haptic perception of location and texture, this group found bilateral haptic texture-selectivity in parietal operculum and posterior visual cortex (Sathian et al., 2011). They also reported bimodal activity in some sites within posterior visual, left inferior frontal cortex, and the MOC. Interestingly, this study did not report any visual or haptic response to texture in the collateral sulcus (as was found by Puce et al., 1996; Peuskens et al. 2004, Cant and Goodale, 2007; and Cant et al., 2009). It should be noted that Stilla and Sathian explored haptic and visual perception of two different groups of object stimuli, one depicting texture, and the other depicting shape. Additionally, they used 3-D representations (3D) of texture and shape for the haptic condition, while using 2-dimensional (2D) photographs of texture and shape for the visual condition. 
In the current imaging study, we examined the brain regions involved in visual and haptic recognition of surface texture and shape. Importantly, however, participants explored physically identical stimuli in both the haptic and the visual conditions. We were primarily interested in whether or not the same medial ventral-stream structures implicated in the visual perception of surface texture are also engaged when people explored the same textures via touch. To do this, we used a block-design fMRI study in which participants attended to either the "shape" or "texture" of objects using "vision only" or "touch only" in a given block. Within each sensory domain, the activation associated with texture blocks was contrasted with that observed in shape blocks, to reveal areas of the brain that were more involved in the perception of object texture through vision, and through haptics. We also carried out the reverse contrast to examine the brain regions involved in visual and haptic processing of object shape. We found that although the same general region of medial occipitotemporal cortex was activated during both haptic and visual texture judgments, the areas of activation were not overlapping between the two sensory modalities. An anterior region of the parahippocampal gyrus (PHG) was activated bilaterally during visual-texture judgments, whereas an adjacent area located more posteriorly was activated during haptic-texture judgments, but only in the left hemisphere, contralateral to the palpating hand. 


\section{$2.2 \quad$ METHODS}

\subsubsection{Participants}

13 right-handed individuals aged 19 to $32(M=27 S E=1 ; 6$ female) with normal or corrected-to-normal vision participated in our study. Informed consent was collected in accordance with the guidelines approved by the University of Western Ontario Health Sciences Research Ethics Board and in accordance with the standards outlined in the 1964 Declaration of Helsinki. Participants were compensated $\$ 25$ for each hour of their time.

\subsubsection{Object Stimuli}

The stimuli used in the present study were a number of real 3D objects made of resin. Two different texture patterns were combined with two different 3D shapes to create four distinguishable object stimuli (see Figure 1a). The first shape was a pyramid and the second shape was a 3D quadrilateral. One texture consisted of rows of indented dots, along all surfaces of the object. The second texture was comprised of rows of indented lines, also present along each face. All shapes were created for this experiment using a hard-white plastic casting resin, thereby ensuring that the color and low-level material properties remained constant across all stimuli. 
The objects were presented in the center of a platform within the scanner, located at a comfortable viewing level just above the abdomen of each participant (see Figure 1b). The workspace was illuminated by a light fixed to the head-coil and by a light inside the bore of the scanner. Efforts were made to ensure that the objects were within a comfortable arm's reach of the right hand of each participant. The orientation of the objects on each trial was varied randomly across five different orientations along the zaxis so that each shape was presented an equal number of times in each orientation across the experiment.

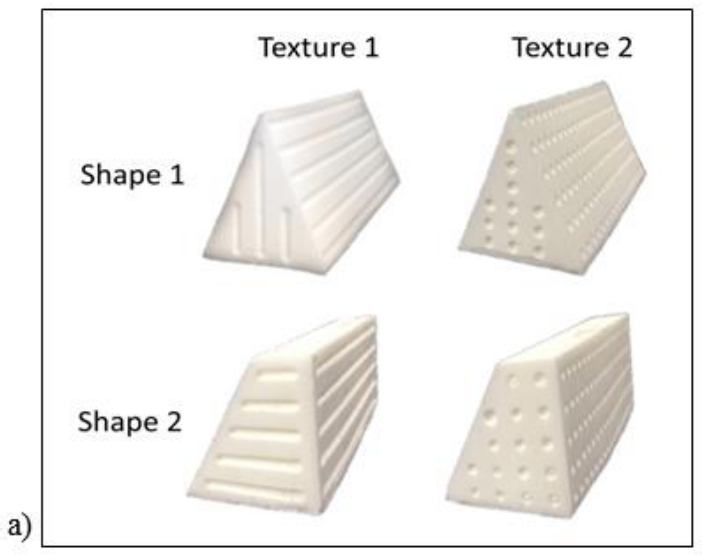

\section{b)}
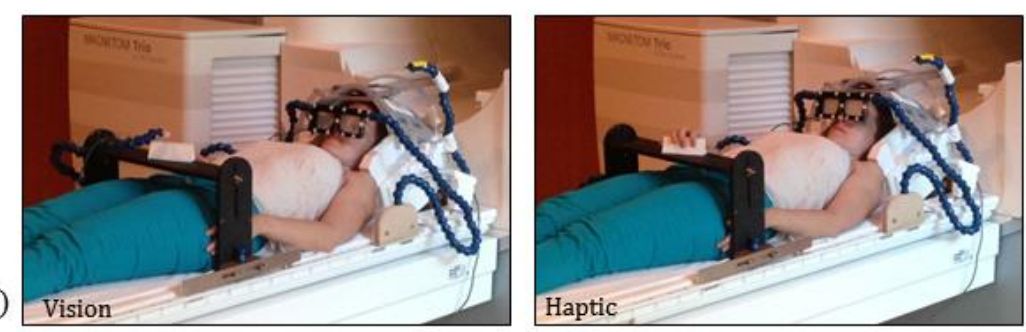

Figure 1a. Two depictions of texture (rows of indented lines and rows of indented dots) were combined with two shape exemplars (pyramid and an asymmetrical quadrilateral) to create the 3D object stimuli used in the experiment. Figure $1 b$. The experimental set up. The photograph on the left shows the setup in the vision condition, where participants lay supine in a head-tilted position, and viewed objects presented to them on a platform located above the waist. Modified LCD PLATO goggles were used to control stimulus 
viewing time (but are not shown here). Particpants maintained fixation upon an LED positioned above the object. The photograph on the right depicts the set up in the haptic condition, which was similar to the visual condition except that the modified LCD PLATO goggles remained closed for the entire run. During the haptic condition, participants made a small rotation of the forearm (though minimal motion overall) in order to palpate objects positioned on the table.

\subsubsection{Experimental Procedure and Design}

We used the same block-design across two different runs; vision runs (where participants examined the three-dimensional objects visually) and haptic runs (where they explored the objects haptically). Within the runs, the objects were presented in two different conditions; shape blocks (where participants were explicitly instructed to attend to the shape of the objects) and texture blocks (where they were instructed to attend to the texture of the objects).

Each scan run started with an auditory cue, instructing participants to either "look" at or "touch" the objects for the entire duration of the run. Another auditory cue was delivered at the onset of each block within a particular run indicating to the participant whether they were to attend to the "texture" or to the "shape" of the proceeding trials of objects.

Each shape and texture block consisted of five pairings of individual object presentations followed by a cued response. Using the 'left' and 'right' keys on an fMRI- 
compatible response-box, participants indicated whether the preceding pair of objects were the same or different with respect to their texture or shape (depending on the given block condition), using the index finger of their left hand.

Objects were presented for $2 \mathrm{~s}$ and were separated by 2 -s rest (see Figure 2). In the texture blocks, the objects varied in their texture, while their shape remained consistent - and vice versa for the shape blocks. In both the vision and haptic runs, blocks started with a 2-s auditory cue, lasted for $50 \mathrm{~s}$, and were separated by $16 \mathrm{~s}$ of baseline. The baseline consisted of a rest condition in which the goggles were closed. In addition, participants were asked to keep their eyes shut when the goggles were closed in order to minimize eye movements outside of the trials.

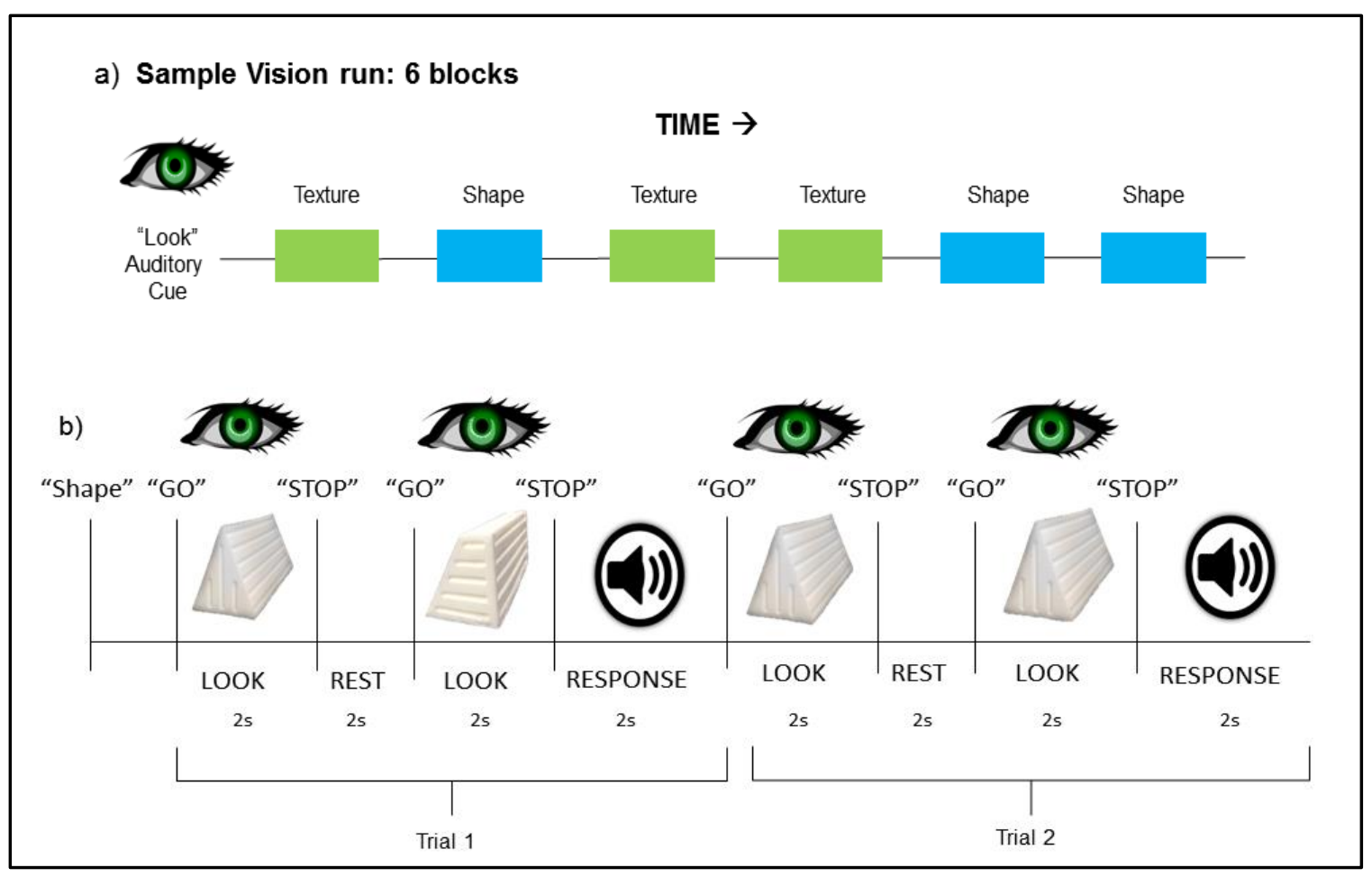

Figure $2 a$. Six blocks in a sample vision run. Half were shape and half were texture blocks. Figure $2 b$. Trial progression within one sample "shape" block. Each block started 
with an auditory cue indicating the block condition. Participants were then presented with object pairings that either varied in their texture or shape - depending on the given block (for $2 \mathrm{~s}$ each) before they made a discrimination response. The figure shows two sample object-pairing trials, however each block consisted of five trials. Shape and texture blocks followed the same timing, as did the haptic and visual runs.

The same block paradigm was used for both vision and haptic runs. In the vision runs, viewing was controlled using modified crystal LCD PLATO goggles (Translucent Technologies: www.translucent.ca/plato). In the haptic condition, the goggles remained closed throughout the entire run, and participants were instructed, by an auditory "go", to move their hand to the table in order explore the object on each trial until they heard an auditory "stop", which instructed them to stop exploring. An identical beep was presented during vision and haptic runs. When exploring the object in the haptic condition, participants were encouraged to use a "natural" palpation pattern and to refrain from moving or lifting the objects. In the vision condition, participants were instructed to fixate on a small red LED light located just above the object on the table.

Run order was counter-balanced within and between observers using a balanced Latin square design (Bradley, 1958) and response button mapping was also counterbalanced across participants. On average, each participant completed five haptic runs and five vision runs. 


\subsubsection{MRI Acquisition}

The experiment was carried out on a 3-Tesla Siemens Magnetom Tim Trio imaging system at the Robarts Research Institute at Western University (London, Ontario, Canada). The functional data was acquired with a $\mathrm{T} 2 *$-weighted single-shot gradient-echo echo-planar imaging sequence with interleaved slice acquisition. Foam padding was used to minimize head motion in the tilted head-coil and a strap was used to minimize upper arm motion. The parameters for obtaining functional data were: field of view $(\mathrm{FOV})=210 \mathrm{~mm} \times 210 \mathrm{~mm}$; in-plane resolution $=3.0 \mathrm{~mm} \times 3.0 \mathrm{~mm}$, slice thickness $=3.5$ (zero gap); 36 axial slices; echo time $(\mathrm{TE})=30$; repetition time $(\mathrm{TR})=$ $2000 \mathrm{~ms}$; flip angle $(\mathrm{FA})=78^{\circ}$. Functional data were aligned to high-resolution anatomical images obtained using a 3D T1-weighted MPRAGE sequence $(\mathrm{TE}=2.98 \mathrm{~ms}$ $\mathrm{TR}=2300 \mathrm{~ms} ; \mathrm{TI}$ (inversion time $)=900 \mathrm{~ms} ; \mathrm{FA}=9^{\circ} ; 192$ contiguous slices of $1 \mathrm{~mm}$ thickness; FOV = $240 \mathrm{~mm} \times 256 \mathrm{~mm}^{2}$ ).

\subsection{5 fMRI Data Pre-processing and Analysis}

The data were pre-processed and analyzed using Brain Voyager QX (Version 1.10.2, Brain Innovation, Maastricht, Netherlands). Following slice scan-time correction, functional data were assessed for head motion and/or magnet artifacts by viewing cineloop animation and examining motion detection parameter plots following 3D motion correction algorithms on the untransformed two-dimensional data, aligned to the functional volume closest in time to the anatomical scan. Data from one participant was 
excluded due to excessive head motion in the haptic runs (greater than $3 \mathrm{~cm}$ within a run), and data from one additional participant was excluded because of severe scanner artifacts in the data. A 6-mm Gaussian kernel was used in the spatial smoothing of the functional images in the runs. Functional data were pre-processed with high-pass temporal filtering to remove frequencies below 3 cycles/run. Functional volumes were then superimposed on anatomical brain images transformed into Talairach space (Talairach and Tournoux, 1998).

\subsection{RESULTS}

\subsubsection{Whole Brain Random Effects Analysis}

Voxel-wise random-effects general linear model (GLM) analyses were performed on the averaged data of 13 participants, separately for the haptic and visual runs. A number of contrasts were run to localize visual and haptic regions that showed selectivity for shape and for texture. For each of the contrasts, the group activation maps were set to a minimum statistical threshold $(p<0.005)$ and minimum cluster size threshold of: 6 functional voxels $\left(\right.$ of $\left.(3 \mathrm{~mm})^{3}\right)$ for the haptic-texture and -shape contrasts, 8 functional voxels for the visual-texture contrast, and 7 functional voxels for the visual-shape contrasts. All corrections were based on Brain Voyager's cluster threshold estimation plug-in, run at $\mathrm{p}<0.05$. The plug-in estimates the spatial smoothness of the maps and computes the threshold accordingly. Since contrast maps may differ in smoothness, they 
may differ in threshold, as was the case here. All corrections yielded the same corrected p value of 0.005 .

In order to localize visually texture-selective regions of the brain, a contrast was performed to isolate areas that showed greater activation when visually perceiving texture than when visually perceiving shape [visual-texture > visual-shape]. Significant activation was found in the right cingulate gyrus (CG), an insular (INS) region of the right hemisphere, along with bilateral activation along four medial regions of the parahippocampal gyrus (or collateral sulcus, CoS) (see Figure 3). We then looked at the data from the haptic runs and ran a contrast to isolate texture-selective regions using a contrast of haptic-texture > haptic shape (see Figure 4). We found activation in the right middle frontal gyrus (MFG), the left insula (INS), and in a dorsal branch of the left posterior collateral sulcus (pCoS), just below the lingual gyrus, when participants were palpating the objects to identify their texture vs. when they were palpating for shape [haptic-texture > haptic-shape] (see table 1). Interestingly, the visual texture-selective regions did not overlap with the haptically texture-selective area. Even at a reduced statistical threshold ( $\mathrm{p}<0.01$ uncorrected) the region of haptic texture activation within the left collateral sulcus lay adjacent to, but not overlapping with the region responsive to visual textures (see Figure 5). 


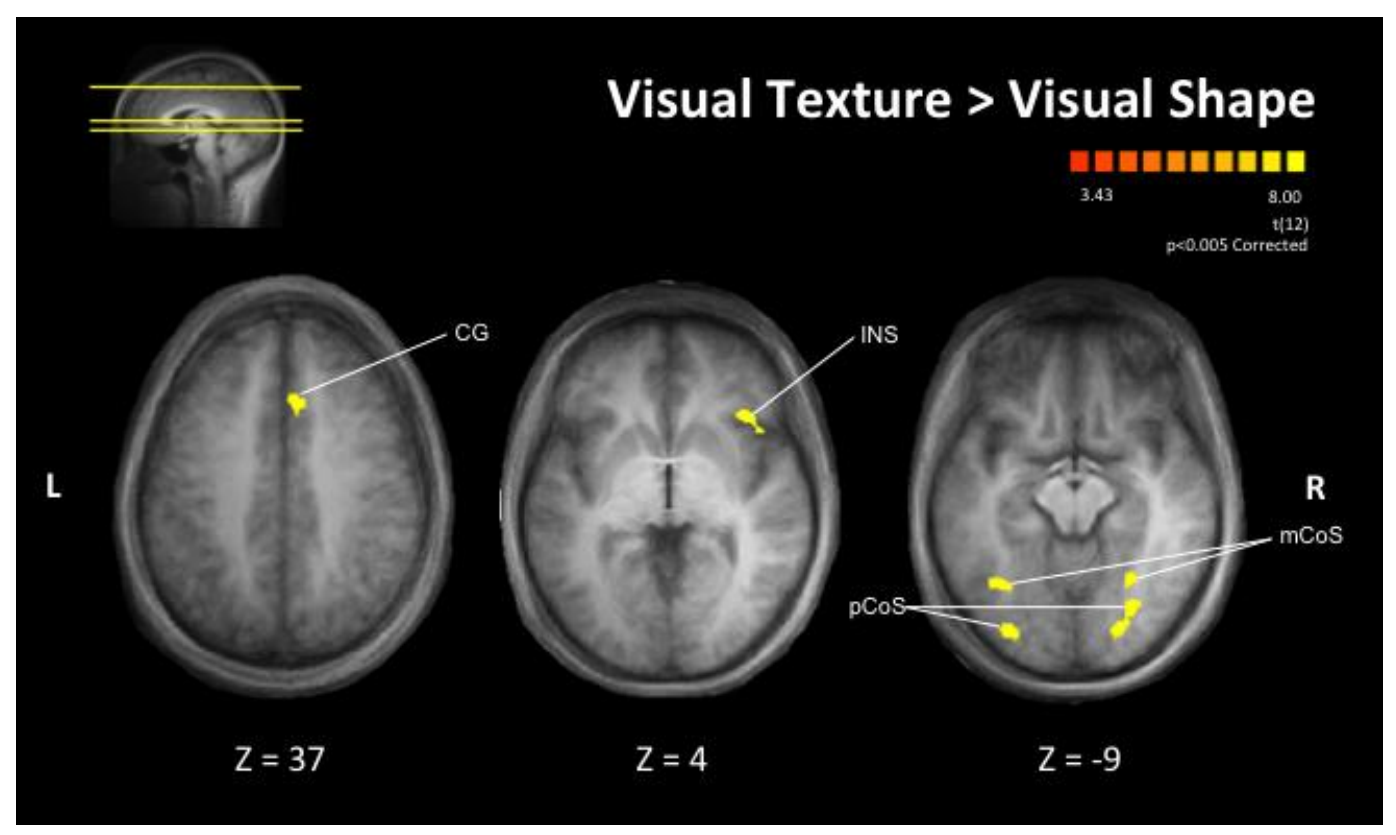

Figure 3. Visually texture-selective areas. A vision [texture $>$ shape] contrast revealed activity in the right cingulate gyrus (CG), right insula (INS), and bilaterally along middle and posterior regions of the collateral sulcus $(\mathrm{mCoS}$ and $\mathrm{pCoS})$. Slice plane levels are depicted in the top left corner, with the most dorsal slice plane reflecting the leftmost frontal longitudinal slice below. 


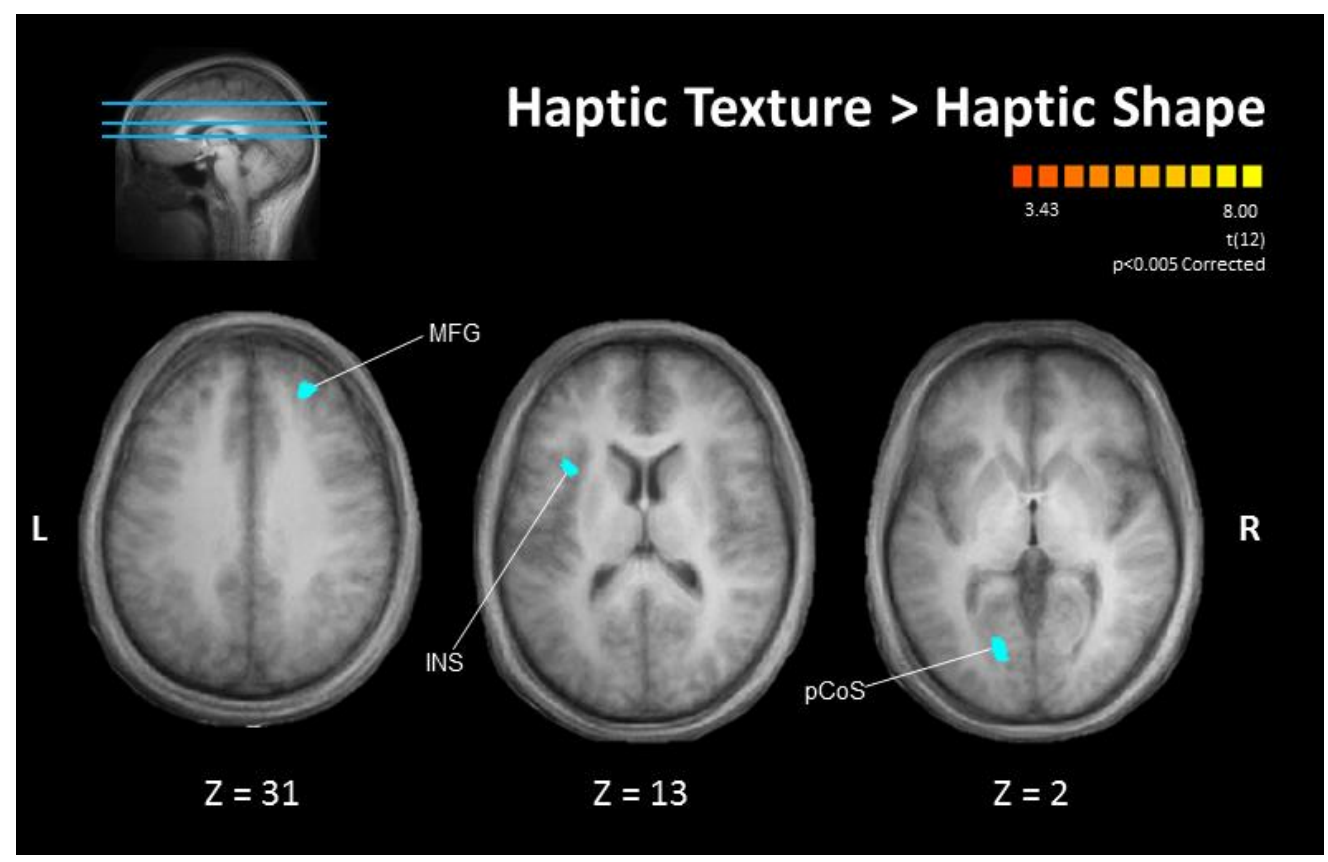

Figure 4. Haptic-texture selective areas. Texture-selective activity was observed in the right middle frontal gyrus (MFG), left insula (INS), and in a posterior region of the left collateral sulcus (pCoS). Slice plane levels are depicted in the top left corner, with the most dorsal slice plane reflecting the leftmost frontal longitudinal slice below.

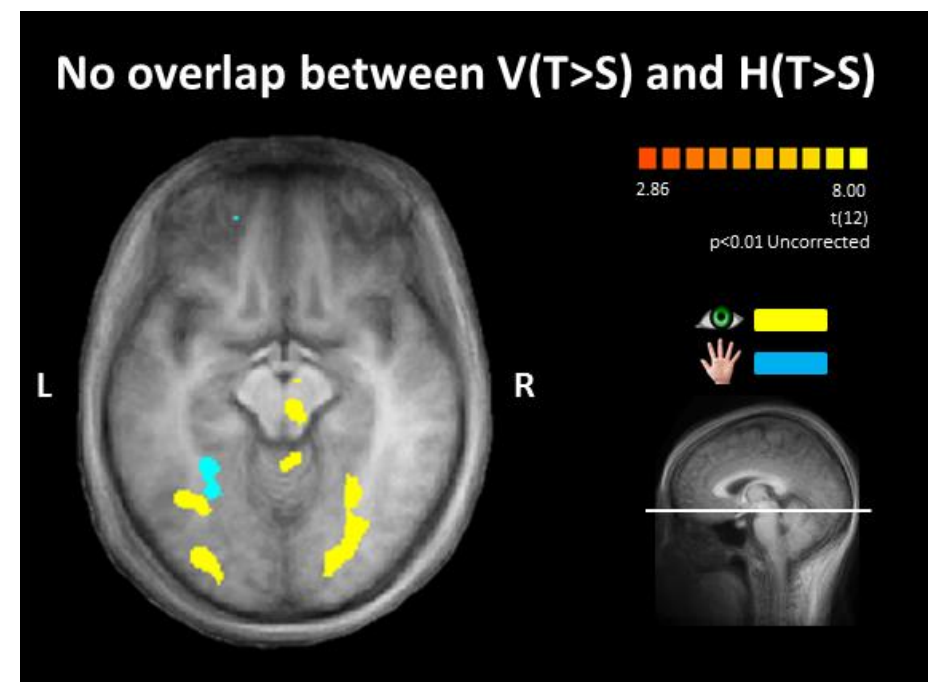

Figure 5. Haptic (shown in blue) and visual (shown in yellow) texture activation at a (relaxed) threshold of $\mathrm{p}<0.01$. More robust visual-texture activity was observed 
bilaterally along the collateral sulcus $(\mathrm{CoS})$. Haptic-texture activation was observed in the left $\mathrm{CoS}$, directly abutting but not overlapping the visual-texture activity. The slice plane is depicted in the lower right corner image.

\section{$\underline{\text { Vision Texture }>\text { Shape }}$}

\begin{tabular}{lccr} 
Area & \multicolumn{3}{c}{ Left Hemisphere } \\
& \multicolumn{2}{c}{ Talairach coordinates } \\
& $x$ & $y$ & $z$ \\
Middle collateral sulcus & -34 & -59 & -9 \\
Posterior collateral sulcus & -31 & -78 & -9 \\
Cingulate gyrus & & & \\
Insula & & &
\end{tabular}

$\underline{\text { Haptic Texture }>\text { Shape }}$

\begin{tabular}{llcccccc} 
Area & \multicolumn{2}{c}{$\begin{array}{l}\text { Left Hemisphere } \\
\text { Talairach Coordinates }\end{array}$} & \multicolumn{3}{c}{$\begin{array}{l}\text { Right Hemisphere } \\
\text { Talairach Coordinates }\end{array}$} \\
& $x$ & $y$ & $z$ & & $x$ & $y$ & $z$ \\
Posterior collateral sulcus & -17 & -68 & 2 & & & \\
$\begin{array}{l}\text { Middle frontal gyrus } \\
\text { Insula }\end{array}$ & -33 & 13 & 15 & & & \\
\end{tabular}

Table 1. Coordinates of the regions that activated for visual-texture (above) and haptictexture (below).

We next ran a contrast to isolate regions that were visually shape-selective [visual-shape > visual-texture] and regions that were haptically shape-selective [hapticshape $>$ haptic-texture]. The visual-shape contrast revealed activation in the secondary somatosensory region (SII) of the LH, the left superior temporal gyrus (STG), left middle temporal gyrus (MTG), and bilaterally in the lateral occipital complex (LOC), in addition to a number of other object processing regions (see table 2). 


\section{Vision Shape $>$ Texture}

\begin{tabular}{|c|c|c|c|c|c|c|}
\hline \multirow[t]{2}{*}{ Area } & \multicolumn{3}{|c|}{$\begin{array}{l}\text { Left Hemisphere } \\
\text { Talairach Coordinates }\end{array}$} & \multicolumn{3}{|c|}{$\begin{array}{l}\text { Right Hemisphere } \\
\text { Talairach Coordinates }\end{array}$} \\
\hline & $x$ & $y$ & $z$ & $x$ & $y$ & $z$ \\
\hline \multirow[t]{3}{*}{ Superior temporal gyrus } & -56 & -43 & 26 & & & \\
\hline & -51 & -56 & 17 & & & \\
\hline & -41 & -72 & 14 & & & \\
\hline Parietal operculum (SII) & -58 & -23 & 22 & 52 & -31 & 22 \\
\hline Early visual & -12 & -88 & 17 & & & \\
\hline Middle temporal gyrus & -43 & -56 & 6 & & & \\
\hline Lateral occipital cortex & -50 & -67 & 2 & 48 & -67 & 2 \\
\hline
\end{tabular}

\section{Haptic Shape $>$ Texture}

\begin{tabular}{|c|c|c|c|c|c|}
\hline \multirow[t]{2}{*}{ Area } & \multicolumn{2}{|c|}{$\begin{array}{l}\text { Left Hemisphere } \\
\text { Talairach Coordinates }\end{array}$} & \multicolumn{3}{|c|}{$\begin{array}{l}\text { Right Hemisphere } \\
\text { Talairach Coordinates }\end{array}$} \\
\hline & $y$ & $z$ & $x$ & $y$ & $z$ \\
\hline Intra parietal sulcus & & & 47 & -38 & 44 \\
\hline
\end{tabular}

Table 2. Coordinates of the regions that activated for visual-shape (above) and hapticshape (below).

The haptic shape [haptic-shape > haptic-texture] contrast revealed a large area of activation in the intraparietal sulcus (IPS) (see figure 6). Overall, however, activation in the haptic condition was less extensive than the vision condition. Surprisingly, however, we did not find significantly greater activation for haptic-shape than for haptic-texture in the LOC. Therefore, we ran a contrast between both conditions (haptic-shape; haptictexture) > baseline and found that the LOC was activated in both the contrast of [hapticshape > baseline] and the contrast of [haptic-texture > baseline]. The LOC was also activated during shape identification in both modalities (vision-shape; haptic-shape) more than during the baseline rest period. These data suggest that both haptic exploration of shape and texture engaged the LOC but to a comparable degree. 


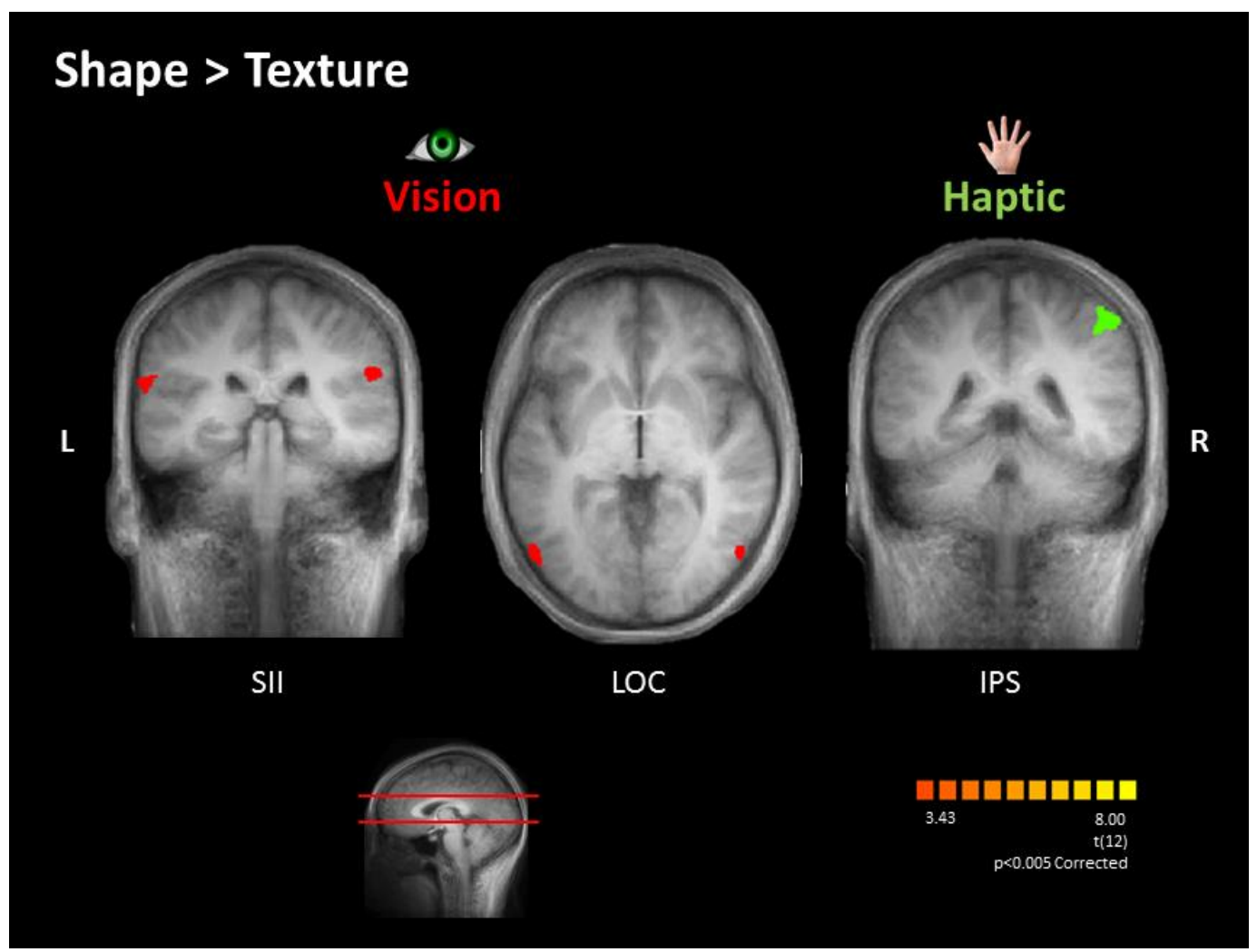

Figure 6. Visual and haptic shape selective areas. A vision [shape > texture] contrast (red) revealed bilateral visual shape-selective activity in somatosensory regions (SII) and in the lateral occipital cortex (LOC) at a threshold of $\mathrm{p}<0.005$. Slice plane levels are depicted below, with the most dorsal slice plane reflecting the leftmost longitudinal LOC slice below. A haptic [shape > texture] contrast (green) revealed right intraparietal sulcus (IPS) activation at $\mathrm{p}<0.005$.

\subsubsection{Group-level ROI analyses}

We next used the brain areas identified in the whole-brain voxel-wise analysis of visual texture vs. shape as independent regions-of-interest to examine whether these areas showed sensitivity to haptic texture vs. shape. In order to determine whether these visual 
areas contained any information about haptic texture, we ran a [haptic-texture $>$ hapticshape] contrast in each visual-texture region. There was no significant $(\mathrm{p}<0.005)$ haptic texture activation (haptic texture > haptic shape) within these visual ROIs in the right CG $(\mathrm{t}=1.90, \mathrm{p}=0.08)$ the right $\mathrm{INS}(\mathrm{t}=0.98, \mathrm{p}=0.35)$ or the $\operatorname{CoS}(\mathrm{t}=0.63, \mathrm{p}=0.54 ; \mathrm{t}=0.65$, $\mathrm{p}=0.53 ; \mathrm{t}=1.14, \mathrm{p}=0.28 ; \mathrm{t}=-0.80, \mathrm{p}=0.44)$.

Conversely, we used the haptic data to isolate regions that showed greater activation to haptic texture than to haptic shape [haptic-texture > haptic-shape] (as in the whole brain RFX analysis above). Again, we did not find any significant visual texture related activation [visual-texture $>$ visual-shape] either in the right $\mathrm{MFG}(\mathrm{t}=-1.0, \mathrm{p}=$ $0.33)$, the left INS $(t=1.75, \mathrm{p}=0.12)$ or in the left $\mathrm{pCoS}(\mathrm{t}=0.24, \mathrm{p}=0.81)$.

In summary, we found greater activation for visual-texture versus visual-shape within medial regions of the collateral sulcus, as expected based on prior literature (Cant and Goodale, 2007; Cant et al., 2009; Cavina-Pratesi et al., 2010). We also found activation in ventral 'visual' areas of the brain, namely the left $\mathrm{pCoS}$, when participants palpated objects for texture rather than shape perception. The areas that showed activation for visual-texture perception lay adjacent to, but did not overlap with, areas that activated during haptic-texture perception. Region-of-interest analyses (which are less conservative because they do not require correction for massive multiple comparisons) confirmed that the visual texture-selective areas did not contain any information about haptic texture, and vice versa for the haptic texture-selective areas. 


\subsection{DISCUSSION}

We used fMRI to investigate whether medial occipitotemporal regions of visual cortex that are known to be involved in the perception of visual object-texture, are also involved in the perception of object-texture via touch.

Importantly, we used an attention modulation paradigm, in conjunction with an adaptation design, in order to encourage participants to attend to the particular object property that was of interest in a given block. The attention modulation paradigm was similar to that used in Cant and Goodale (2007) whereby participants' attention was directed to the object property of interest (either texture or shape) by varying this on each trial. The fMR-adaptation component was added by repeating the unattended property which presumably resulted in a reduced HRF with successive repetitions.

A group-level RFX GLM analysis revealed visually texture-selective regions bilaterally in two middle regions of the collateral sulcus (mCoS) and two more posterior regions of the collateral sulcus ( $\mathrm{pCoS})$, consistent with the results of previous imaging studies (Cant et al., 2007; 2009 ; Cavina-Pratesi et al., 2010). These findings suggest that medial areas of occipitotemporal cortex play a role in the visual encoding object surface properties. Visual attention to object texture also activated regions of the right cingulate gyrus (CG) and right insula (INS). 
We were particularly interested in determining whether the medial regions of the collateral sulcus involved in the visual processing of texture were also activated when participants explored the same texture exemplars haptically. Haptic perception of texture was not observed in any of the visually texture-selective regions. Rather, a more dorsal posterior region of the left $\mathrm{CoS}$ was activated when participants explored texture via touch. Sub threshold haptic-related activation in the anterior collateral sulcus was located in a region directly adjacent to, but not overlapping with, activation observed during visual texture perception. Taken together, these results suggest that the regions of the brain that are processing visual texture cues are spatially distinct from those involved in processing haptic texture cues.

That the areas of activation for haptic and visual texture did not overlap in the collateral sulcus may speak to the fact that the information coded by haptic and visual representations of object-texture can be somewhat different. For example, one can see that a surface is shiny without perceiving it as being sticky. The perception of "stickiness" occurs only when you reach out and feel that surface. Additionally, a long history of behavioral research suggests that at least for perceiving surface texture, bimodal processing is no better than unimodal (Guest and Spence, 2003; Bjorkman, 1967; Lederman and Abbott, 1981; Heller, 1982; Picard, 2007).

At the same time, the proximity of the haptic and visual activation also suggests that they may be closely linked. After all, it has been argued that the visual perception of a particular surface property typically invokes an earlier association with haptic 
exploration of that surface (Cant and Goodale, 2007). In fact, we rarely perceive texture by touch alone. More often, touch perception is accompanied, if not motivated by, initial visual perception (Schifferstein and Cleiren, 2005). For instance, when we see a feathered surface, we may perceive "softness" - a construct that is based on prior experience in which we actually felt "softness." Furthermore, work by Lacey et al. (2010) suggests that multisensory representations of surface properties such as texture likely include information from modality-independent representations. In this sense, learned associations between earlier visual and haptic experiences may facilitate the binding of haptic and visual information about object texture. It may be the case that the haptic activation seen here in what are typically regarded as visual regions of the brain may reflect the binding of haptic and visual object properties that aid in recognition - and learned associations between the visual appearance of objects and their material properties.

The same argument can be made for the relationship between acoustic and somatosensory information about objects. Arnott et al. (2008), for example, reported that one region within the right anterior parahippocampal cortex was activated when people listened to sounds associated with the handling of different materials, such as paper and tinfoil. However, a more posterior region of the parahippocampal cortex was activated during visual-material property perception. These results suggest that different areas within occipitotemporal cortex are specialized to represent material properties based on the sensory domain through which these properties are either directly perceived or invoked by learned associations (Arnott et al., 2008). 
Both the visual- and haptic-texture contrasts resulted in activation of nonoverlapping regions of the middle temporal gyrus (MTG), cingulate gyrus (CG), and insula (INS). The cingulate gyrus activity may reflect a number of task-related components such as error detection or online monitoring of performance (Carter et al, 1998), which were introduced by the discrimination task used in our study. Although both shape and texture information were present on every trial, participants were explicitly instructed to attend to and make a judgment relative to only one object property (i.e., shape or texture), while ignoring the other. We also observed texture-selective activation within the INS. Sathian et al. (2011) also found texture-selectivity in the INS and noted reports that somatosensory responses to tactile stimuli activate the INS in monkeys (Robinson and Burton, 1980), although they acknowledged that more work was required to determine the actual role of the INS in texture perception. Again, one can speculate that the visual texture-related activations in the INS reflect learned associations with haptic texture perception.

The occurrence of activity in traditionally visual regions of the brain during haptic texture perception also raises the question that imagery may be driving the activation seen during haptic runs. The issue of imagery in haptic perceptual tasks has long been debated; however studies exist to support the fact that though imagery may be involved, it is not a necessary component of haptic perception (Sathian et al., 1997; Merabet et al., 2007). A study by Newman et al. (2005) examined the role of visual imagery of objects on cortical activity and found that the same brain network activated when people imagined objects as when they perceived them, and that imagery did not enhance cortical 
activity. The haptic-texture activation we observed is unlikely to be due to visual imagery. If participants were imagining visual representations of the textures while palpating them, then we would expect to see activation in areas overlapping the visual texture-selective ones. On the contrary, we found that the regions that were activated during texture perception did not overlap with visual areas. Additional evidence against an imagery explanation stems from the fact that haptic activity was located solely in the left hemisphere, contralateral to the palpating hand-whereas an imagery explanation might predict a bilateral response.

It is worth noting that attention modulation (ie. directing attention to a particular property) does not rule out the possibility that participants may have experienced involuntary processing of the unattended stimulus property. This possibility highlights the differences (and overlap) between attention, perception, and discrimination. Though all three processes would likely have been involved in this task, because we averaged the activity across a period of time when all three would have been occurring, the results of this experiment do not tease apart the regions that are involved in each process separately. It is up to future research to determine when attention ends and when perception begins.

The current results speak to the fact that at there is no overlap between haptic and visual texture selective regions in the brain at a gross anatomical level. It is important to note that more specific, multivariate methods such as multi-voxel 
pattern analysis could unveil some fine-grained inter-digitation between the senses at a sub-voxel level.

We also examined shape selectivity, as compared to texture, in both the visual and haptic domains. Visual shape perception activated bilateral shape-selective lateral occipital cortex (LOC) (Kourtzi \& Kanwisher, 2001) in addition to a number of regions of the left hemisphere including: the superior temporal gyrus (STG), early visual regions, and MTG. Interestingly, we also found visual-shape selective activation in bilateral regions of secondary somatosensory cortex (SII) within the parietal operculum.

Haptic-shape selectivity resulted in activation in the right intraparietal sulcus (IPS), a region related to somatosensory shape representations (Bodegard et al., 2000; Stoesz et al., 2003; Miquee et al., 2007), but failed to activate shape-selective LOC (Amedi et al., 2001; 2002). LOC has been found to encode texture information and since both the [haptic-texture > rest] and [haptic-shape > rest] contrasts revealed LOC activation, it is likely that LOC was encoding aspects of haptic texture in addition to haptic shape. Haptic texture encoding in LOC may have been enhanced by the stimuli used. Our exemplars of texture were designed to have a fixed, and therefore somewhat unnatural, linear pattern to them which may have incorporated a slightly geometric shape-like consistency to the texture that activated the LOC.

In summary, we found that both haptic and visual perception of surface texture activates medial occipitotemporal cortex, although this activity is located in spatially 
distinct regions. This finding suggests that texture is encoded differently for vision and touch, and that regions of occipitotemporal cortex may be specialized to underlie these unique sensory representations. Nevertheless, the close proximity of these areas of activation suggests that they may be functionally linked. 


\subsection{REFERENCES}

Allen, H.A. and Humphreys, G.W. (2009). Direct tactile stimulation of dorsal occipitotemporal cortex in a visual agnosic. Current Biology, 19(12) 1044 - 1049.

Amedi, A., Malach, R., Hendler, T., Peled, S., and Zohary, E. (2001). Visuo-haptic

object- related activation in the ventral visual pathway. Nature Neuroscience, 4:324-330

Amedi, A., Jacobson, G., Hendler, T., Malach, R., and Zohary, E. (2002). Convergence of visual and tactile shape processing in the human lateral occipital complex. Cereb Cortex, 12:1202-1212.

Amedi, A., Von Kriegstein, K., Van Atteveldt, N., Beauchamp, M.S., and Naumer, M.J. (2005). Functional imaging of human cross modal identification and object recognition. Experimental Brain Research 166: 559-571.

Arnott, S.R., Cant, J.S., Dutton, G.N., and Goodale, M.A. (2008). Crinkling and crumpling: An auditory fMRI study of material properties. NeuroImage. 43, 368378.

Bjorkman, M. (1967). Relations between intra-modal and cross-modal matching, Scand. J. Psychol., 8, 65-76.

Bodegard, A., Ledberg, A., Geyer, S., Naito, E., Zilles, K., and Roland, P.E. (2000). Object shape differences reflected by somatosensory cortical activation. $J$. Neurosci., RC51.

Bradley, J.V. (1958) Complete counterbalancing of immediate sequential effects in a Latin square design. Journal of the American Statistical Association, 53: 525-528.

Cant, J.S. and Goodale, M.A. (2007). Attention to form or surface properties modulates different regions of human occipitotemporal cortex. Cereb. Cortex, 17:713-731. 
Cant, J.S., Arnott, S.R., and Goodale, M.A. (2009). fMR-adaptation reveals separate processing regions for the perception of form and texture in the human ventral stream. Exp. Brain Res, 192:391- 405.

Carter, C.S., Braver, T.S., Barch, D.M., Botvinick, M.M., Noll, D., and Cohen, J.D. (1998). Anterior cingulate cortex, error detection, and the online monitoring of performance. Science, 280 (5364).

Cavina-Pratesi, C., Kentridge, R.W., Heywood, C.A., and Milner, A.D. (2010). Separate channels for processing form, texture, and color: Evidence from fMRI adaptation and visual object agnosia. Cerebral Cortex, 20 (10), 2319-2332.

Grefkes, C., Weiss, P.H., Zilles, K., and Fink, G.R., (2002). Cross modal processing of object features in human anterior intraparietal cortex, an fMRI study implies equivalencies between humans and monkeys. Neuron 35, 173-184.

Grill-Spector, K. and Malach, R. (2004).The Human Visual Cortex. Annual Reviews Neuroscience, 27: 649-677.

Guest, S. and Spence, C. (2003). What role does multisensory integration play in the visuotactile perception of texture? Int. J. Psychophysiol., 50, 63-80.

Heller, M.A. (1982). Visual and tactual texture perception: Intersensory cooperation Percept. Psychophys., 31, 339-344.

James, T.W., Humphrey, K., Gati, J.S., Servos, P., Menon, R.S., and Goodale, M.A. (2002). Haptic study of three-dimensional objects activates extrastriate visual areas. Neuropsychologia, 40, 1706-1714.

Kaas, J.H. and Collins, C.F. (2003). The organization of somatosensory cortex in anthropoid primates. Adv. Neurol., 93, 57-67. 
Kourtzi, Z. and Kanwisher, N. (2001). Representation of perceived object shape by the human lateral occipital complex. Science, 293, 1506-1509.

Lacey S., Tal N., Amedi A., and Sathian K. (2009). A putative model of multisensory object representation. Brain Topogr. 21, 269-274.

Lacey, S., Flueckiger, P., Stilla, R., Lava, M., and Sathian, K. (2010). Object familiarity modulates the relationship between visual object imagery and haptic shape perception. Neuroimage, 49, 1977-1990.

Lederman, S.J. and Abbott, S.G. (1981). Texture perception: studies of intersensory organization using a discrepancy paradigm, and visual versus tactual psychophysics. J. Exp. Psychol. Hum. Percept. Perform., 7, 902-915.

Lucan, J.N., Foxe, J.J., Gomez-Ramirez, M., Sathian. K., and Molholm, S. (2010). Tactile shape discrimination recruits human lateral occipital cortex during early perceptual processing. Hum. Brain Mapp., 11, 1813-21.

Merabet, L.B., Swisher, J.D., McMains, S.A., Halko, M.A., Amedi, A., Pascual-Leone, A., and Somers, D.C. (2007). Combined activation and deactivation of visual cortex during tactile sensory processing. J. Neurophysiol., 97:1633-164.

Miquee, A., Xerri, C., Rainville, C., Anton, J.-L., Nazarian, B., Roth, M., and ZennouAzogui, Y. (2007). Neuronal substrates of haptic shape encoding and matching: A functional magnetic resonance imaging study. Neurosci., 152 (1), 29-39.

Naumer, M.J., Ratz, L., Yalachkov, Y., Polony, A., Doehrmann, O., van de Ven, V., Muller, N.G., Kaiser, J., and Hein, G. (2010). Visuohaptic convergence in a corticocerebellar network. (2010). European Journal of Neuroscience, 1-7. 
Nelson, A.J., Staines, W.R., Graham, S.J., and McIlroy, W.E. (2004). Activation in SI and SII: the influence of vibrotactile amplitude during passive and task-relevant stimulation. Brain Res. Cogn. Brain Res., 19, 174-184.

Newman, S.D., Klatzky, R.L., Lederman, S.J., and Just, M.A. (2005). Imagining material versus geometric properties of objects: an fMRI study. Cogn. Brain Res., 23, 235246.

O'Sullivan BT, Roland PE, and Kawashima R. (1994). A PET study of somatosensory discrimination in man. Microgeometry versus macrogeometry. Eur. J. Neurosci. $137-148$.

Peuskens, H., Claeys, K.G., Todd, J.T., Norman, J.F., Van Hecke, P., and Orban, G.A. (2004). Attention to 3-D shape, 3-D motion, and texture in 3-D structure from motion displays. J. Cognit. Neurosci., 16:665-682.

Picard, D. (2007). Tactual, visual, and cross-modal transfer of texture in 5- and 8-yearold children. Perception, 36, 722-736.

Pietrini, P., Furey, M.L., Ricciardi, E., Gobbini, M.I., Wu, W.H.C., Cohen, L., Guazzelli, M., and Haxby, J. (2004). Beyond sensory images: object-based representation in the human ventral pathway. Proc Natl Acad Sci., 101:5658-5663.

Puce, A., Allison, T., Asgari, M., Gore, J.C., and McCarthy, G. (1996). Differential sensitivity of human visual cortex to faces, letterstrings and textures: a functional magnetic resonance imaging study. J. Neurosci., 16:5005-5215.

Robinson, C.J. and Burton, H. (1980). Somatic submodality distribution within the second somatosensory (SII), 7b, retroinsular, postauditory and granular insular cortical areas of M. fascicularis. J Comp Neurol.192:93-108. 
Sathian, K., Zangaladze, A., Hoffman, J.M., and Grafton, S.T. (1997). Feeling with the mind's eye. NeuroReport. 8:3877-3881.

Sathian, K., Lacey, S., Stilla, R., Gibson, G.O., Deshpanae, G., Hu, X., LaConte, S., and Glielmi, C. (2011). Dual pathways for haptic and visual perception of spatial and texture information. Neuroimage, 57, 462-475.

Schifferstein, H.N.J. and Cleiren, M.P.H.D. (2005). Capturing product

experiences: a split-modality approach. Acta Psychol. (Amst.), 118, 293-318.

Servos, P., Lederman, S., Wilson, D., and Gati, J. (2001). fMRI-derived cortical maps for haptic shape, texture and hardness. Cognit. Brain Res. 12:307-313.

Simões-Franklin, C., Whitaker, T. A., and Newell, F. N. (2011). Active and passive touch differentially activate somatosensory cortex in texture perception. Hum. Brain Mapp. 32, 1067-1080.

Stilla, R. and Sathian, K. (2008). Selective visuo-haptic processing of shape and texture. Hum. Brain Mapp. 29:1123-1138.

Stoesz, M.R., Zhang, M., Weisser, V.D., S.C., Prather, Mao, H., and Sathian, K. (2003). Neural networks active during tactile form perception: common and differential activity during macrospatial and microspatial tasks. Int J Psychophysiol., 50, 4149.

Tal, N. and Amedi, A. (2009). Multisensory visual-tactile object related network in humans: insights gained using a novel cross modal adaptation approach. Exp Brain Res., 198 (2-3): 165-182.

Tanaka, J., Weiskopf, D., and Williams, P. (2001). The role of color in high-level vision. Trends in Cognitive Sciences, 5, 211-215. 
Talairach, J. and Tournoux, P. (1988). Co-planar stereotaxic atlas of the human brain. Thieme Medical Publishers.

Zhang, M., Weisser, V.D., Stilla, R., Prather, S.C., and Sathian, K. (2004) Multisensory cortical processing of object shape and its relation to mental imagery. Cogn Affect Behav Neurosci, 4:251-259. 


\section{Chapter 3}

\section{General Discussion}




\subsection{GENERAL DISCUSSION}

\subsubsection{Summary of findings}

The visual and haptic perception of texture resulted in activation of separate cortical regions that, even at a relaxed statistical threshold, were neighboring but not overlapping. Consistent with previous literature, the visual perception of shape activated regions of the lateral occipital cortex (LOC), whereas the visual perception of texture activated more medial regions, including the posterior branch of the collateral sulcus (CoS) (Peuskins et al., 2004; Cant and Goodale, 2007; Cant et al., 2009).

Additionally, when we isolated shape-selective areas in the haptic condition using a contrast between shape and baseline-rest, we found activation in LOC, confirming that the haptic-shape task recruited the LOC. Since there have been reports of textureencoding within the LOC (Cant and Goodale, 2007) we also ran a contrast between haptic-texture and baseline-rest, and found that the LOC was activated during haptic texture exploration. These results suggest that both texture and shape information are encoded in the LOC when stimuli 3D are perceived via touch.

\subsubsection{Haptic and visual processing of object texture}

The visually texture-selective regions in our study were located in very close proximity to the haptic texture-selective regions, though interestingly, there was no 
overlap between these two modality-specific regions. This suggests that visual representations of texture might be encoded somewhat differently from haptic representations.

Whitaker et al., (2008) suggested that during the perception of familiar objects (as opposed to novel or abstract shapes), visual and haptic processing of a given texture may be more likely to be integrated (and by extension - to show greater overlap in activation in occipitotemporal cortex). We chose to use abstract stimuli that would minimize any influence of top down/semantic processing in promoting overlap of the visual and haptic regions, and consequently did not see any overlap.

Further support for distinct representations of texture in vision and haptics comes from behavioral evidence suggesting that bimodal perception of texture does not enhance accuracy of perception when compared with unimodal perception - unlike in visuo-haptic form perception, in which bimodal presentation has been shown to enhance performance. Work by Lederman and Abbott (1981) showed that bimodal processing of texture was not superior when compared to unimodal visual vs. tactile processing of the same texture. A study by Jones and O’Neill (1985) also demonstrated that accuracy in the bimodal perception of texture was no better than accuracy when texture was perceived through each sense alone, and the decision speed in the bimodal condition was the average between vision alone and haptic alone. Lastly, work by Guest and Spence (2003) found that visual perception was affected by haptic tasks when participants were making 
discriminations about texture, whereas tactile performance on the same discrimination task was unaffected by visual information.

This behavioral evidence supports the notion that visual and haptic texture processes are likely to be somewhat independent. In line with Whittaker et al.'s (2008) viewpoint, our results suggest that visual representations of texture are encoded by different neural populations from haptic representations of texture.

Visual- and haptic-texture activation is located in close proximity on the CoS, indicating the likelihood of a link between the representations in each modality. We rarely perceive the texture of an object through vision or haptics alone. Rather, the representation of texture in one sense is linked to the representation in the other. For instance we may visually perceive a surface as looking "rough" or "coarse" -descriptions that heavily rely on a haptic experience of the word, which is based on prior experience with a "rough" or "coarse" surface. Is it likely that the neural regions which underlie haptic- and visual-texture perception are located in close proximity because they facilitate a communicatory relationship between these two modalities.

Within the haptic and visual domains, the conceptualization of shape has been shown to be similarly represented based on categorization judgements (Gaissert and Wallraven, 2012), and has also been shown to activate bimodal regions of shape-selective LOC (Amedi et al., 2002). The fact that haptic texture-selective regions are not bimodal in the same way that haptic-shape regions are, suggests that there may be a difference 
between the way in which humans perceive texture through vision as compared to touch. The close proximity of visual- and haptic-texture activation, however, suggests a fundamental link between the way the visual and haptic processing of texture.

\subsubsection{Evidence for separate pathways for haptic surface texture vs shape processing}

Within the haptic domain, we found that different regions of the brain were involved in texture perception and in shape perception (although the LOC was only activated in the haptic contrasts against baseline, as discussed further below). Haptic texture perception activated the left posterior $\operatorname{CoS}$, the left insula (INS), and the right middle frontal gyrus (MFG). Haptic shape perception activated the right intraparietal sulcus (IPS). In vision, texture perception activated bilateral regions of the middle and posterior $\mathrm{CoS}$, the right cingulate gyrus (CG), and the right INS. Visual shape perception activated regions of the left superior and middle temporal gyrus (STG; MTG), the left early visual cortex, and bilateral secondary somatosensory cortex (SII) and LOC. These results suggest that as in vision, the haptic perception of shape and surface texture is likely divided across two separate cortical pathways.

Our results support the division of labor between haptic encoding of texture and shape suggested by James et al. (2007). James et al. (2007) argued that haptic perception of object properties involves a divergent path whereby geometric surface properties are processed predominantly within higher-level somatosensory area SII, whereas geometric 
shape properties are processed in multimodal areas including the LOC and aIPS. Interestingly, James et al., (2007) suggested that there may be more multisensory visuohaptic activity in shape-selective regions, as compared to texture-selective regions of the brain. More research is required to determine the degree to which visual vs. haptic texture-selective regions of the brain may inform one another.

\subsubsection{Relation of the findings to previous research on visual form and texture recognition}

In the vision condition, we found shape-selective activation in the LOC and texture-selective activation in regions of the middle- and posterior CoS. This lateralshape vs. medial-texture encoding was expected in light of prior research (Peuskens et al., 2004; Cant and Goodale, 2007; Cant et al., 2009; Cavina-Pratesi et al., 2010).

Surprisingly, haptic perception of shape (versus texture) did not selectively activate the LOC, as we had hypothesized - based on earlier work by Amedi et al. (2001; 2002). This result could be due to the nature of our stimuli; participants in this study palpated real 3D textured shapes to extract texture cues, which may have led to the recruitment of the LOC during both the form and texture tasks. Consistent with this hypothesis, the LOC was activated during the form and texture tasks versus the baseline rest condition. It may be the case that it was more difficult for participants to ignore texture and attend to shape (and vice versa) during haptic exploration (Klatzky et al., 1987) than during the visual task. 
Further, the textures of our stimuli were not naturalistic, but were comprised of linear patterns, and therefore may have elicited shape-based processing. We used linear textures in order to ensure that the orientation of both stimulus shape and texture could be varied across trials. In other words, one of the goals of the experiment was to equate as much as possible between both the shape and texture conditions, along with the haptic and visual conditions.

Finally, a study by Reed et al. (2004) found that LOtv was more activated by "real" objects than by meaningless objects. Because our study involved stimulus exemplars that were controlled for shape and texture dimensions, they were meaningless objects without any explicit high-level semantic properties, which may account for the reduction in haptic shape activity in LOtv.

\subsubsection{Conclusions}

In the present study, haptic-texture activation in the collateral sulcus was neighboring but not overlapping with the visual-texture activation. Haptic object perception is encoded by two different streams (similar to visual encoding), that are specialized for processing texture information and shape information, within ventral regions of the brain. We found that regions of the left CoS, right MFG, and left INS were more involved in the haptic perception of texture than shape. The right IPS was found to be more involved in haptic perception of shape than texture. Haptic-shape regions overlapped with visual-shape activation (when the shape contrast was relative to 
baseline), suggesting that haptic texture was perhaps processed involuntarily along with shape. Given the lack of evidence for overlapping (possibly bimodal) texture-selective brain regions, it is likely that haptic and visual representations of texture are considerably different, particularly in comparison to haptic and visual representations of shape. 


\subsection{REFERENCES}

Amedi, A., Malach, R., Hendler, T., Peled, S., and Zohary, E. (2001). Visuo-haptic object-related activation in the ventral visual pathway. Nature Neurosci. 4:324330.

Amedi, A., Jacobson, G., Hendler, T., Malach, R., and Zohary, E. (2002). Convergence of visual and tactile shape processing in the human lateral occipital complex. Cereb Cortex., 12:1202-1212.

Cant, J.S. and Goodale, M.A. (2007). Attention to form or surface properties modulates different regions of human occipitotemporal cortex. Cereb. Cortex.,17:713731.

Cant, J.S., Arnott, S.R., and Goodale, M.A.(2009). fMR-adaptation reveals separate processing regions for the perception of form and texture in the human ventral stream. Exp. Brain Res., 192:391-405.

Cavina-Pratesi, C., Kentridge, R.W., Heywood, C.A., and Milner, A.D. (2010). Separate channels for processing form, texture, and color: Evidence from fMRI adaptation and visual object agnosia. Cerebral Cortex, 20 (10), 2319-2332.

Gaissert, N. and Wallraven, C. (2012). Categorizing natural objects: a comparison of the visual and the haptic modalities. Experimental Brain Research 216(1) 123-134.

Guest, S. and Spence, C. (2003). What role does multisensory integration play in the visuotactile perception of texture? Int. J. Psychophysiol. 50, 63-80.

James, T.W., Kim, S., and Fisher, J.S. (2007). The neural basis of haptic object processing. Can Journ of Exp Psych. 61 (3), 219-229. 
Jones, B. and O’Neill, S. (1985). Combining vision and touch in texture perception. Percept. Psychophys. 37, 66-72.

Klatzky, R.L., Lederman, S.J., and Reed, C.L. (1987). Theres more to touch than meets the eye: the salience of object attributes for haptics with and without vision. $J$. Exp. Psychol. Gen. 116, 356-369.

Lederman, S.J. and Abbott, S.G. (1981). Texture perception: studies of intersensory organization using a discrepancy paradigm, and visual versus tactual psychophysics. J Exp Psychol Hum Percept Perform. 7, 902-915.

Peuskens, H., Claeys, K.G., Todd, J.T., Norman, J.F., Van Hecke, P., and Orban, G.A. (2004). Attention to 3-D shape, 3-D motion, and texture in 3-D structure from motion displays. J. Cognit. Neurosci., 16:665-682.

Reed, C.L., Shoham, S., and Halgren, E. (2004) Neural substrates of tactile object recognition: an fMRI study. Hum Brain Mapp., 21:236- 246.

Whitaker, T.A., Simoes-Franklin, C., and Newell, F.N. (2008). Vision and touch: Independent or integrated systems for the perception of texture? Brain Research, $59-72$. 


\section{SAMANTHA PODREBARAC, MSc}

Masters in neuroscience student with extensive project management and fMRI research experience (design, execution, and analysis).

\section{EDUCATION/AFFILIATIONS}

Sept 2011 - Present $\quad$ Masters of Science, Graduate program in neuroscience, Western University, Canada.

Advisor: Professor Melvyn Goodale

Sept 2007 - April 2011 Honors Specialization in Psychology, BS.c. Western University, Canada. Thesis: Gender-selective neural populations: evidence from eventrelated. fMRI repetition suppression.

Advisors: Professor Melvyn Goodale \& Dr. Jacqueline C. Snow

\section{EMPLOYMENT}

May 2011 - Aug 2012 Paid research assistant. Goodale lab. Centre for Brain and Mind, Western University, London Ontario.

Scheduled participants for fMRI and psychophysical experiments, designed psychophysical experiments, programmed fMRI and psychophysical experiments, collected and analysed behavioural and fMRI data, wrote my undergraduate thesis for formal publication, conducted various administrative tasks.

May 2011 - Aug 2012 Paid research assistant. Culham Lab. Centre for Brain and Mind, Western University, London Ontario.

Designed and testing participants on a psychophysical experiment, assisted with the fMRI data collection, conducted various administrative tasks.

Sept 2008 - Feb 2011 Winners Inc., London Ontario.

Cashier/customer service representative. Jewellery counter sales clerk, warehouse stock processing, fitting room attendant, and clothing floor assistant.

June 2007 - Sept 2007

May 2008 - Sept 2008 Receptionist. Toromont Industries (A Caterpillar Equipment Dealership and Repair Centre), Hamilton Ontario.

Main line reception, switchboard control, directed all incoming calls, work order completion, daily bank deposit processing and payroll. 
$\underline{\text { PUBLICATIONS }}$

Podrebarac, S., Goodale, M., and Snow, J. (2013). Are visual texture-selective areas recruited during haptic texture discrimination? Neuroimage. Submitted.

Podrebarac, S., Goodale, M., Van der Zwan, R., \& Snow, J. (2013). Gender-selective neural populations: evidence from event-related. fMRI repetition suppression. Experimental Brain Research, 226 (2), 241-252.

\section{TALKS AND PRESENTATIONS}

May 2013 Poster: Podrebarac, S., Goodale, M.A., and Snow, J.C. (2013). Are visual texture-selective areas recruited during haptic texture discrimination? Vision Sciences Society: 13th Annual Meeting, Naples, Florida, USA, and 7th Annual Canadian Neuroscience Meeting, Toronto, ON, Canada.

May 2012 Talk: Podrebarac, S., Goodale, M., Van der Zwan, R., \& Snow, J. (2012). Gender-selective neural populations within the occipital and fusiform face-areas: Evidence from rapid event-related fMRI. Vision Sciences Society: $12^{\text {th }}$ Annual Meeting, Naples, Florida, USA.

April 2011 Poster: Podrebarac, S. and Snow, J. (2011). The neural representation of gender in human cortex. Senior honors undergraduate thesis poster presentation

Dec 2010 Talk: Leaders forum, Western University. Undergraduate speaker (Topic: Teamwork in research).

\section{TEACHING EXPERIENCE/LEADERSHIP}

Jan 2013 - April 2013 Neuroimaging of cognition (graduate course)

Teaching assistant - also conducted a weekly lab/tutorial section

Sept 2013 - April 2013 Mentored two undergraduate research assistants who worked on my thesis project.

Sept 2012 - Dec 2012 Psychology of sex differences in human brain and behavior. Teaching assistant.

Sept 2011 - April 2012 Introduction to psychology. Teaching assistant.

Sept 2011 - April 2012 Mentored an undergraduate research assistant in the Culham lab.

Sept 2010 - April 2011 Exam proctor. Introduction to psychology.

Sept 2010 - April 2011 Mentored a second year undergraduate volunteer research assistant who was involved with participant testing for my BSc thesis projects. 


\section{RESEARCH EXPERIENCE}

May 2009 - Dec 2011 Volunteer research assistant. Hayden Personality and Emotion Development Lab.Western University. Data collection and entry for a study investigating the role of genetic and environmental factors in childhood cognitive development and psychopathology. Testing methods include: child observation, cortisol sampling and parent self-reports.

Aug 2009 - Sep 2010 Volunteer research assistant. Health Psychology Lab,Western University. Recruitment and testing for a study investigating the recovery effects of pre-operative strength training in knee replacement patients.

Jan 2010 - April 2010 Volunteer research assistant. Hoaken Lab, Western University Testing, data collection and video coding for a study examining the psychological variables predicting poker play outcomes and pathological gambling patterns in undergraduate students.

\section{TECHNICAL PROFICIENCY}

Languages: $\quad$ E-Prime

Software: $\quad$ fMRI, Brain Voyager

Statistical analysis: $\quad$ SPSS, Excel, Numbers (Mac).

Image manipulation: Photoshop

Word processing: $\quad$ Microsoft Word, Powerpoint, Pages \& Keynote (Mac).

\section{AWARDS RECEIVED}

University of Western Graduate Research Scholarship

University of Western Ontario Entrance Scholarship of Excellence

Public speaking award from the Lions club of Ontario

\section{VOLUNTEER EXPERIENCE}

Sept 2010 - May 2011 Surgery waiting room volunteer at University Hospital.

Directed incoming surgical patients to their respective preparatory areas and serving as a liaison between post-operative patients and their family members.

\section{May 2011- Aug2011 \&}

April 2009 - Aug2010 Emergency room volunteer at University Hospital.

Provided information at the main emergency room entrance for visitors and patients, escorted patients to examination rooms, directed visitors in the ER, delivered medication, assembled patients' charts. 
Jan 2010 - Feb 2010 Presidential campaign member for a candidate in the UWO elections. Campaign advertising, promotions and event planning. 